\title{
EMISSION-LINE STARS IN THE OUTER PART OF THE ORION STAR-FORMING REGION
}

\author{
SUHARDJA D WIRAMIHARDJA \\ Department of Astronomy and Bosscha Observatory, Bandung \\ Institute of Technology, Indonesia \\ MAKOTO NAKANO \\ Department of Earth Science, Oita University, Japan \\ AND \\ TOMOKAZU KOGURE \\ Bisei Astronomical Observatory, Bisei-cho, Okayama, Japan
}

\section{INTRODUCTION}

In a series of emission-line star survey programs we have conducted wide and deep survey observations for $\mathrm{H}_{\alpha}$ emission stars in the Orion starforming region. The first result for the Kiso area A-0904 $\left(5^{\circ} x 5^{\circ}\right.$, centered at $\alpha=5^{\mathrm{h}} 40^{\mathrm{m}}$ and $\delta=+0^{\circ}$ ) was presented in Paper I (Wiramihardja et al., 1989), followed by Paper II (Kogure et al., 1989) for the area A$0903\left(\alpha=5^{\mathrm{h}} 20^{\mathrm{m}}, \delta=-5^{\circ}\right.$ and A-0976 $\left(\alpha=5^{\mathrm{h}} 40^{\mathrm{m}}, \delta=-5^{\circ}\right)$. In Paper IV the results for the areas A-1047 $\left(\alpha=5^{\mathrm{h}} 20^{\mathrm{m}}, \delta=-10^{\circ}\right.$ and A-1048 $\left(\alpha=5^{\mathrm{h}} 40^{\mathrm{m}}, \delta=-10^{\circ}\right)$ are given.

Here we present a preliminary result obtained for the Kiso areas A$0831\left(\alpha=5^{\mathrm{h}} 20^{\mathrm{m}}, \delta=+5^{\circ}, \mathrm{A}-0832\left(\alpha=5^{\mathrm{h}} 40^{\mathrm{m}}, \delta=+5^{\circ}\right), \mathrm{A}-0833(\alpha=\right.$ $6^{\mathrm{h}} 00^{\mathrm{m}}, \delta=+5^{\circ}, \mathrm{A}-0902\left(\alpha=5^{\mathrm{h}} 00^{\mathrm{m}}, \delta=+0^{\circ}\right), \mathrm{A}-0905\left(\alpha=6^{\mathrm{h}} 40^{\mathrm{m}}, \delta=\right.$ $+0^{\circ}$, and A-0974 $\left(\alpha=5^{\mathrm{h}} 00^{\mathrm{m}}, \delta=+5^{\circ}\right)$, defined by us as the outer part of the Orion star-forming region.

\section{OBSERVATIONS}

For the whole works of objective-prism observations we used mainly the Kiso $(105 / 150 / 330 \mathrm{~cm})$ Schmidt telescope, and partly the CTIO Curtis $(61 / 91 / 213 \mathrm{~cm})$ Schmidt telescope, with dispersions of $700 \mathrm{Amm}^{-1}$ at $\mathrm{H}_{\alpha}$, respectively. The plate-filter combinations were $103 \mathrm{aE}+\mathrm{RG} 610$ for Kiso and IIIaF + RG630 for CTIO. However, for the present areas, the spectral observations were carried out by the Kiso Schmidt only. In all cases the direct-image plates were collected with the Kiso Schmidt telescope with 
plate-filter combination of IIaD and GG495. All emulsions were hypersensitized by baking in forming gas before exposures.

\section{RESULTS}

More than 12 plates with exposure times between 10 and 120 minutes were inspected visually by magnifiers to detect $\mathrm{H}_{\alpha}$ emission-line stars. The $\mathrm{H}_{\alpha}$ emission strength relative to the adjacent continuum was estimated by eyes into five grades of 5 (very strong), 4 (strong), 3 (medium), 2 (weak) and 1 (very weak). In this way we could detect $4,3,6,3,12$ and $20 \mathrm{H}_{\alpha}$ emission stars in the areas A-0831, A-0832, A-0833, A-0902, A-0905 and A-0974, respectively.

The celestial coordinates of the detected $\mathrm{H}_{\alpha}$ emission stars were measured using the XY machine of the Kiso Observatory. More than 30 SAO stars were adopted as standard stars which give the accuracy better than $2^{-}$for each coordinate. Measurements of the $\mathrm{V}$ magnitudes were carried out with the iris photometer of Kiso Observatory, adopting the stars of Andrews (1981) as standards. The accuracy of the measurements is about $0.1 \mathrm{mag}$ with the limiting magnitude around $\mathrm{V}=17$.

The apparent magnitudes of the detected $\mathrm{H}_{\alpha}$ emission stars are roughly from $\mathrm{V}=13$ to $\mathrm{V}=17$, as in previous papers, suggesting that they are probable candidates of $T$ Tauri type stars, if we adopt a distance of $450 \mathrm{pc}$ to Orion complex, and assume the interstellarabbsorption of $A_{v} \cong 1$ mag. For this outer part of the region, no trend of emission strength is seen. The values of surface densities of emission stars are $0.2,0.1,0.2,0.1,0.5$ and 0.8 per square degrees, for areas A-0831, A-0832, A-0833, A0902, A-0905 and A-0974, respectively. The outer part of the region with these small surface densities probably makes up the boundary of the Orion star-forming region.

The full-length paper is in preparation to be Paper V.

\section{References}

Andrews, A.D., 1981, A Photometric Atlas at the Orion Nebula, Armagh Observatory, Armagh, Northern Ireland

Kogure, T., Yoshida, S., Wiramihardja, S.D., Iwata, T., Ogura, K., 1989, Publ. Astron. Soc. Japain, 41, 1195.

Wiramihardja, S.D., Kogure, T., Yoshida, S., Nakano, M., Ogura, K., Iwata, T., 1991, ibid., 43, 27.

Wiramihardja, S.D., Kogure, T., Yoshida, S., Ogura, K., Nakano, M., 1989, ibid., 41, 155.

Wiramihardja, S.D., Kogure, T., Yoshida, S., Ogura, K., Nakano, M., 1993, ibid., 45, 613. 